\title{
Rhinoscleroma with Rosai-Dorfman Histiocytosis: A Case Report
}

\author{
Anuja S Kulkarni ${ }^{1}$, Prabodh Karnik ${ }^{2}$
}

\begin{abstract}
Rhinoscleroma is a well-documented entity. However, rhinoscleroma associated with lymphadenopathy is a rare clinical entity. Here, we report a case of rhinoscleroma with cervical lymphadenopathy of Rosai-Dorfman histiocytosis in a 57-year-old female patient who presented to us with a history of nasal obstruction of 1-year duration and neck swelling of 2 months duration along with constitutional symptoms such as malaise. Keywords: Cervical lymphadenopathy, Rhinoscleroma, Rosai-Dorfman histiocytosis.

International Journal of Head and Neck Surgery (2018): 10.5005/jp-journals-10001-1347
\end{abstract}

\section{Case Description}

A 57-year-old female patient came with a history of nasal obstruction since 1 year and neck swelling since 2 months. Additionally, the patient complained of recurrent nasal discharge, anosmia, and malaise. Local examination revealed bilateral hypertrophied inferior turbinates and polyps in bilateral nostrils. There was bilateral cervical lymphadenopathy involving multiple lymph nodes on anterior as well as posterior triangles of the neck. Lymph nodes were painless and freely mobile (Fig. 1).

The following specific investigations were done:

- Computed tomography (CT) scan paranasal sinuses.

- Fine-needle aspiration cytology (FNAC) from cervical lymph nodes followed by lymph node biopsy.

- Diagnostic nasal endoscopy and biopsy from nasal cavity mass.

CT scan paranasal sinus revealed nodular enhancing soft tissue in bilateral anterior nasal cavities and multiple enlarged necrotic cervical lymph nodes bilaterally from levels I-IV, VI, and VII, with enlarged level V lymph node on the right side (Fig. 2).

Diagnostic nasal endoscopy revealed polyps in the nasal cavity, firm, rubbery inconsistency, painful, and did not bleed on touch (Fig. 3). Subsequently, a biopsy of nasal polyps was done. It revealed histological features that were suggestive of rhinoscleroma (Fig. 4).

The lymph node FNAC was inconclusive. Hence, lymph node biopsy was done. This revealed features of Rosai-Dorfman histiocytosis (Fig. 5).

Subsequently, the patient received a combination of oral rifampicin (450 mg) and fluoroquinolone levofloxacin $(500 \mathrm{mg}$ ) once a day, for a period of 12 weeks. Within a few weeks of starting the treatment, the patient had symptomatic relief for nasal blockage and visible regression of cervical lymphadenopathy (Fig. 6). After this, oral prednisolone was added in the treatment regime tapering over 4 weeks. Upon which, the cervical lymphadenopathy has regressed significantly.

\section{Discussion}

Rhinoscleroma is a chronic granulomatous infection caused by Klebsiella rhinoscleromatis. ${ }^{1}$ This disease was recognized as an inflammatory process by Mikulicz in 1877 who described the foamy cells which carry his name. Initially, it was considered as

\footnotetext{
1,2Department of ENT and Head Neck Surgery, Jagjivan Ram Hospital, Mumbai, Maharashtra, India
}

Corresponding Author: Anuja S Kulkarni, Department of ENT and Head Neck Surgery, Jagjivan Ram Hospital, Mumbai, Maharashtra, India, e-mail: dr.anujakulkarni@gmail.com

How to cite this article: Kulkarni AS, Karnik P. Rhinoscleroma with Rosai-Dorfman Histiocytosis: A Case Report. Int J Head Neck Surg 2018;9(3):106-109.

Source of support: Nil

Conflict of interest: None

the lesion of the nose alone, but later, other sites of this infection were described. The disease occurs worldwide with low incidence but it is endemic in certain parts of central Europe, North Africa, and Central America. In fully developed infection, the histological appearance is characterized by Mikulicz cells, Russell bodies, and Gram-negative bacteria, but in initial stages, especially, the inflammatory reaction is nonspecific and can be difficult to diagnose. The disease runs in three stages: atrophic, nodular or granulomatous, and cicatricial stages. In the initial stage, the patient complains of rhinorrhea, crusting, foul-smelling discharge, and anosmia. In the second stage, granulomatous nodules cause symptoms of recurrent nasal blockage and stuffiness. In the final stage, fibrosis results in stenosis of nasal vestibule, larynx, or distal airways. Diagnosis can be made on clinical grounds by the stage and pathologically by biopsy demonstrating pseudo epitheliomatous hyperpiesia (a benign proliferation of the superficial epithelium into irregular squamous strands extending down into the deeper epithelial layers) Russell bodies (deposits of gamma globulin seen as small spherical hyaline bodies in cancerous and inflammatory growth and in degenerating plasma cells), and Mikulicz cells (large, round or oval vacuolated phagocyte with small pyknotic nucleus). Treatment consists of prolonged therapy with streptomycin or fluoroquinolones or tetracycline. Local therapy in the nose can consist of nasal irrigation to prevent crusting and reduce rhinorrhea. Surgical intervention for stenosis may be required on occasion.

Rosai-Dorfman histiocytosis also referred as Rosai-Dorfman disease (RDD) is an uncommon benign disorder of unknown 

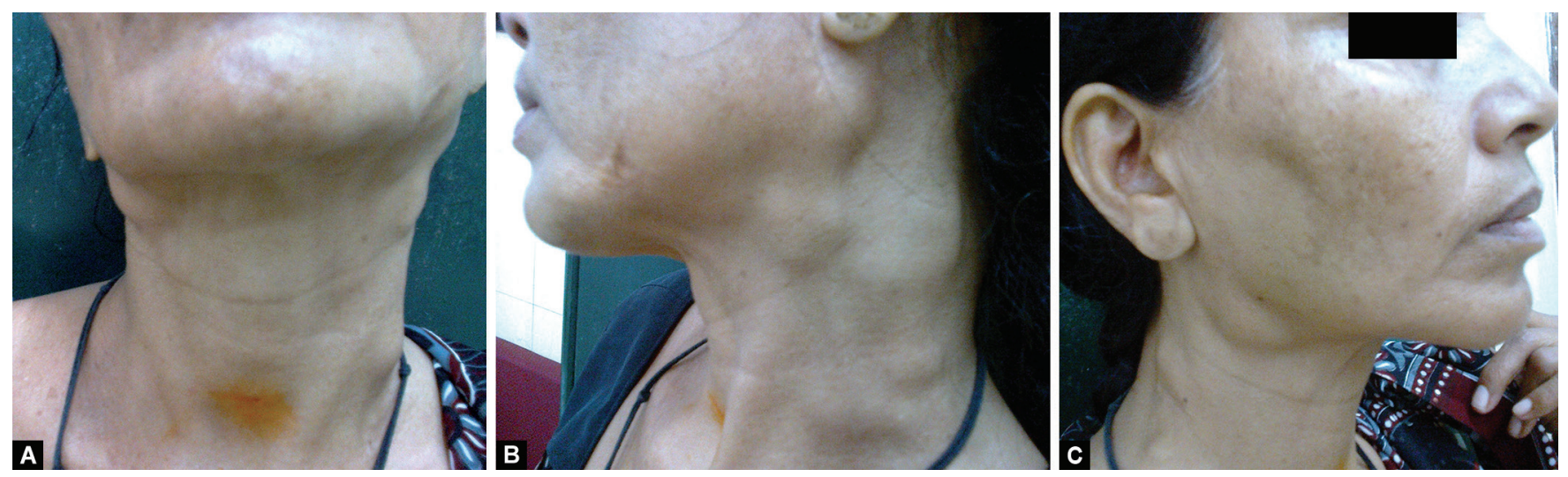

Figs $1 \mathrm{~A}$ to $\mathrm{C}$ : Clinical photograph of a patient depicting bilateral cervical lymphadenopathy involving multiple lymph nodes on anterior as well as posterior triangles of the neck
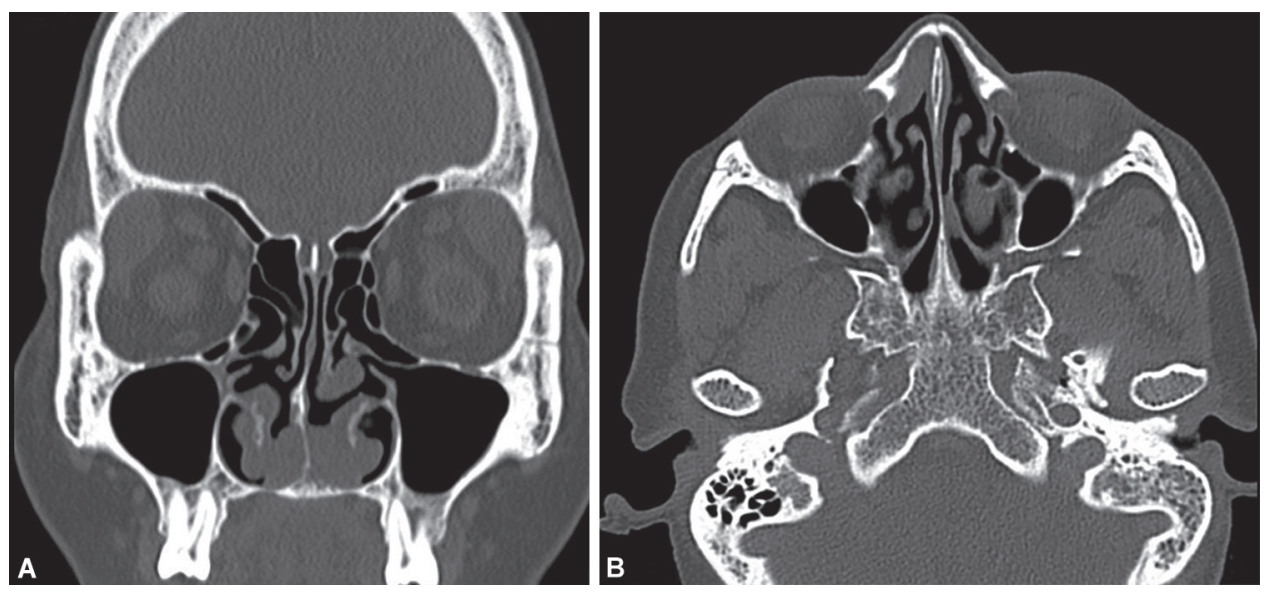

Figs $2 \mathrm{~A}$ and B: CT scan paranasal sinus showing nodular enhancing soft tissue in bilateral anterior nasal cavities (post nonionic contrast, slice thickness $1.5 \mathrm{~mm}$, axial section)

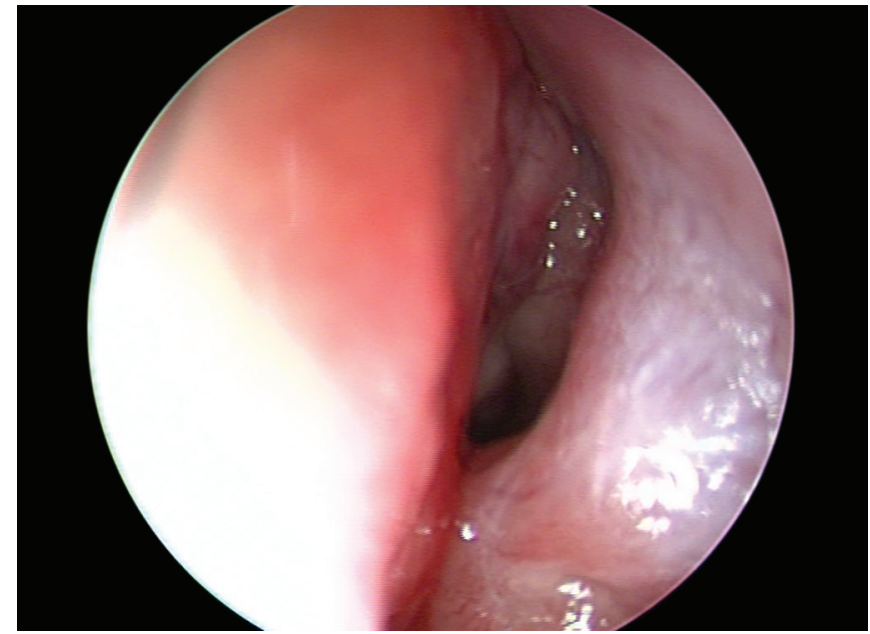

Fig. 3: Diagnostic nasal endoscopy revealing polyps in nasal cavity, firm, rubbery inconsistency, painful, did not bleed on touch

etiology that is characterized by abundant histiocytes in lymph nodes throughout the body. ${ }^{2,3}$ The condition has been named after Ronald F Dorfman and Juan Rosai. It is a nonprogressive disease and self-limited. The patient develops lymphadenopathy of the neck which is the commonest site for histiocyte accumulation. Extra nodal involvement is well recognized and includes several organs, including respiratory tract, skin, nasal cavity, orbit, and bone. The main symptoms presented are massive bilateral painless cervical lymphadenopathy with fever, night sweats, and weight loss, laboratory features suggestive of leukocytosis, elevated erythrocyte sedimentation rate (ESR), and polyclonal hypergammaglobulinemia.

The clinical course of RDD is unpredictable with episodes of exacerbation and remissions that could last many years. The disease is often self-limiting with a very good outcome. RDD patients can be subdivided into three categories: (1) patients with only lymph nodes that enlarge suddenly with spontaneous regression and without any further recurrences; (2) patients with immunologic abnormalities at presentation who have a more widespread nodal disease and a higher fatality rate; (3) patients with several extra nodal site involvement, multimodal disease, and a protracted clinical course with multiple relapses and remissions for years. In these cases, the severity of the disease depends on the type and the number of extra nodal sites.

In the majority of cases, RDD has a benign course and treatment is not necessary. Therapy is required, however, for patients with extra nodal RDD having vital organ involvement or those with nodal disease-causing life-threatening complications. Surgery is generally limited to biopsy, but debulking may be required in patients with vital organ compromise such as intracranial dural-based lesions or upper airway obstruction. When complete resection has excessive morbidity, then partial resection with adjuvant radiosurgery can be successful. Surgical 

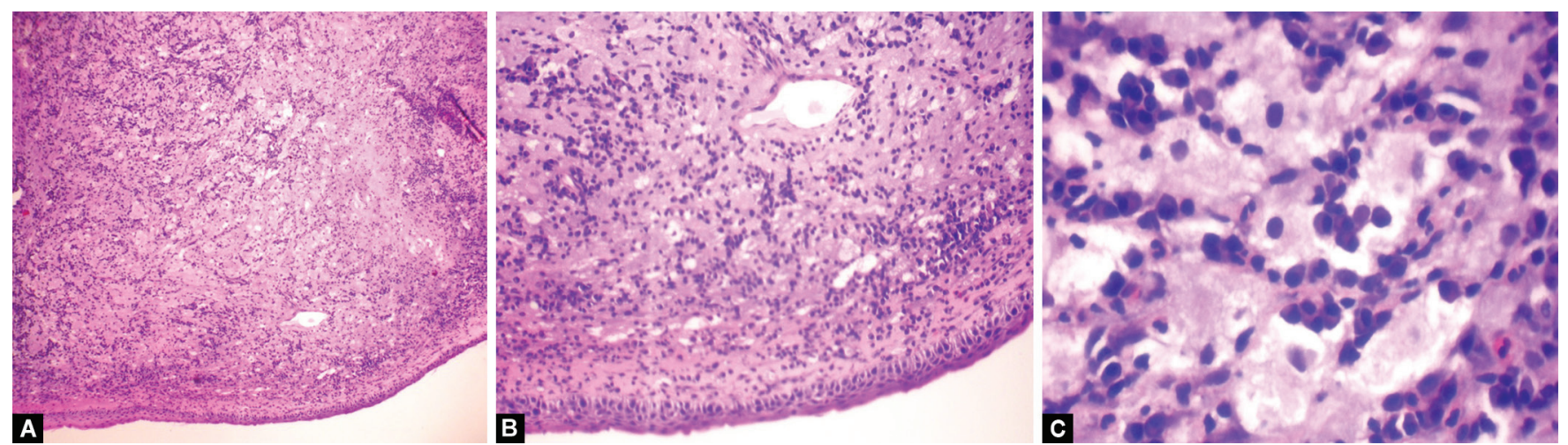

Figs 4A to C: Photomicrograph of histological appearance of biopsy from nasal lesions demonstrating features consistent with rhinoscleroma; (A) 10X magnification; (B) 40X magnification (note Russell bodies and Mikulicz cells); (C) 100X magnification

excision of resectable lesions induced complete remission per literature survey. ${ }^{4}$ Radiotherapy has limited efficacy in most cases, although one recent report showed benefit in a refractory orbital RDD with visual disturbances. ${ }^{5}$ Systemic corticosteroids are usually helpful in the decreasing nodal size and symptoms; however, they can be quite immunosuppressive and recurrence of RDD lesions can occur after a short period of interruption. A prolonged course of low-dose prednisone was very effective in the case of RDD with skin and lymph nodal involvement with respiratory obstruction. ${ }^{6}$

Per literature survey, RDD itself is a very rare clinical entity. The presence of cervical lymphadenopathy in rhinoscleroma is a
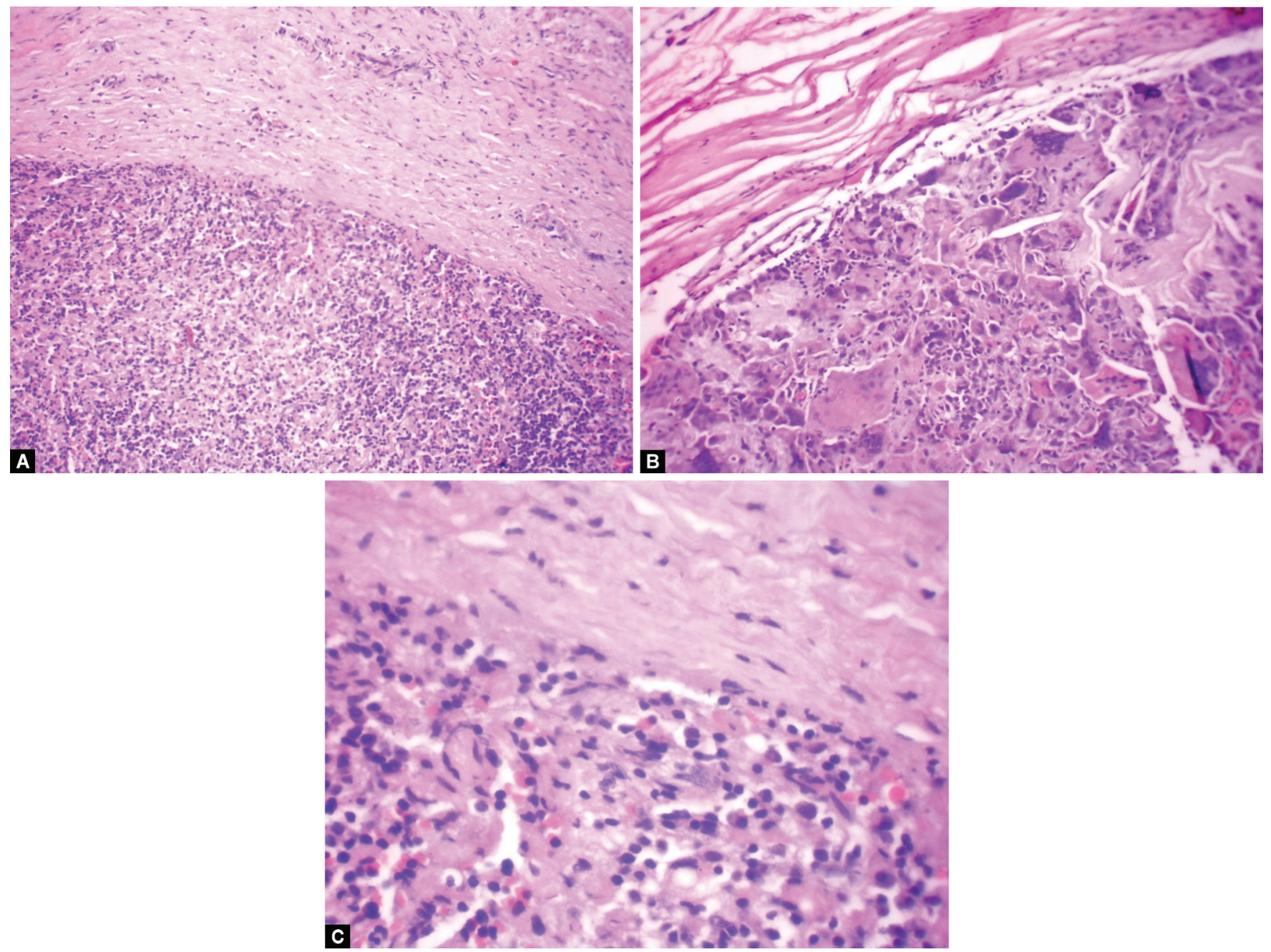

Figs $5 \mathrm{~A}$ to C: Photomicrograph of lymph node biopsy showing nodular infiltrates showing pale staining histiocytes with chronic inflammatory cells in fibrotic background; (A) 10X magnification; (B) 40X magnification; (C) 100X magnification 

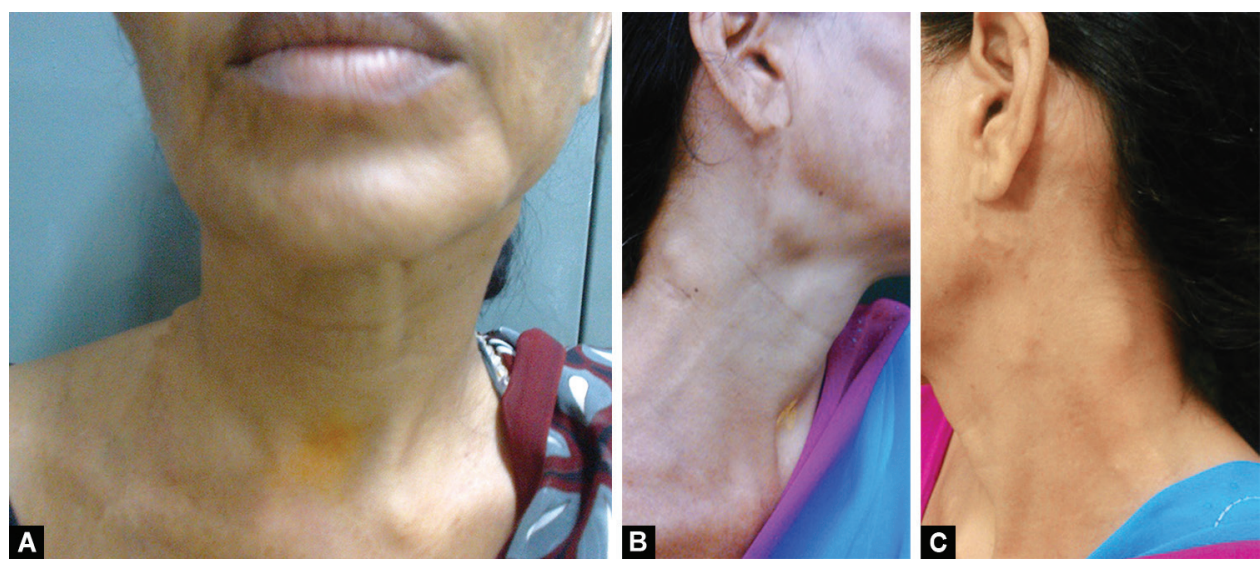

Figs 6A to C: Clinical photograph of patient after medical line of management depicting visible regression of cervical lymphadenopathy; (A) At four weeks; (B) At eight weeks; (C) At twelve weeks

very rare occurrence. So far, only two such cases of coincidence of rhinoscleroma with RDD have been reported in the literature. ${ }^{7}$

In the present case, the patient received a combination of oral rifampicin $(450 \mathrm{mg})$ and fluroquinolone levofloxacin (500 mg), for a period of 12 weeks. Within a few weeks of starting the treatment, the patient had symptomatic relief for nasal blockage and visible regression of cervical lymphadenopathy. Thus, this indicates that lymphadenopathy of Rosai-Dorfman histiocytosis could be secondary to an immunological response to rhinosclerosis.

\section{Conclusion}

Rhinoscleroma and RDD is a rare occurrence. As in the present case, treatment for rhinoscleroma by fluoroquinolones and rifampicin resulted not only symptomatic relief for rhinoscleroma but also resulted in visible regression of cervical lymphadenopathy, thus, indicating that lymphadenopathy of RDD could be secondary to immune response to rhinosclerosis (common antigen leading to these two conditions). There is a room for further research in this rare entity.

\section{References}

1. Cannady Steven B, Citardi Martin J, et al. Granulomatous Diseases of the Nose and Paranasal Sinuses. In. de-Souza C, Har-el G. ed. Head and Neck Surgery, 1st ed., Jitendar P. Vij, India: Jaypee Brothers Medical Publishers(P), Ltd.; 2009. p. 367, vol. 1, ch. 26.

2. Rosai J, Dorfman RF. Sinus histiocytosis with massive lymphadenopathy. Arch Pathol 1969;87:63-70.

3. Foucar E, Rosai J, et al. Sinus histiocytosis with massive lymphadenopathy (Rosai-Dorfman disease): review of the entity. Semin Diagn Pathol 1990;7(1):19-73. PMID 2180012, //www.ncbi.nlm.nih. gov/pubmed/2180012.

4. Pulsoni A, Anghel G, et al. Treatment of sinus histiocytosis with massive lymphadenopathy (Rosai-Dorfman disease): report of a case and literature review. Am J Hematol 2002;69:67-71. DOI: 10.1002/ajh.10008.

5. Cooper SL, Chavis PS, et al. A case of orbital Rosai-Dorfman disease responding to radiotherapy. J Pediatr Hematol Oncol 2008;30: 744-748. DOI: 10.1097/MPH.0b013e31817e4ac1.

6. Oka M, Kamo T, et al. Successful treatment of Rosai-Dorfman disease with low-dose oral corticosteroid. J Dermatol 2009;36:237-240. DOI: 10.1111/j.1346-8138.2009.00630.x.

7. Naraghi M, Madani SZ, et al. Coincidence of Rhinoscleroma and Rosai-Dorfman Disease (sinus histiocytosis with massive lymphadenopathy): report of a case. Acta Med Iran 2002;40(1):11-15. 